Tech Insight

\title{
High-throughput profiling of posttranslational modification enzymes by phage display
}

\author{
Michelle Yen and Jun Yin \\ Department of Chemistry, The University of Chicago, Chicago, IL, USA
}

Phage display has been used as a high-throughput platform for identifying proteins or peptides with desired binding or catalytic activities from a complex proteome. Recently, phage display has been applied to profile the catalytic activities of posttranslational modification (PTM) enzymes. Here, we highlight recent work elucidating the downstream targets of PTM enzymes by phage display, including the genome-wide profiling of biosynthetic enzymes subject to phosphopantetheinyl transferase (PPTase) modification.

\section{Introduction}

Phage display provides a high-throughput platform for functional profiling of peptide or protein libraries based on their binding or catalytic activities. To construct a phage-display library, randomized DNA encoding short peptides (for peptide library assembly) and cDNA or genomic DNA libraries from an organism (for protein library assembly) are cloned into the phage genome or a phagemid vector as fusions to the phage capsid gene (1). Upon expression of the fusion gene, the foreign peptide or protein is covalently anchored on the phage surface as part of the phage capsid. Thus, a phage-display library comprises a collection of phage clones, each containing a different fusion and displaying a unique protein on its surface. The library is then subjected to iterative rounds of selection to substantially enrich for phage particles displaying targets with the desired binding or catalytic functions. Finally, the identities of the selected peptides or proteins are determined by sequencing the fusion genes in the enriched population. Thus far, phage display has been used to select peptides with high-binding affinities for target receptors (2), for the identification of peptides as the substrates of enzyme catalysis $(3,4)$, and for the evolution of catalytic antibodies or enzymes with novel or more efficient catalytic activities (5-10). Recently, we and others have applied phage display to profile the catalytic activities of posttranslational modification (PTM) enzymes $(11,12)$. Here, we discuss phage display as an approach for high-throughput identification of the downstream proteins modified by PTM enzymes.

\section{Protein Posttranslational Modification}

Protein PTM plays an important role in controlling the structure, function, subcellular localization, and degradation of the modified proteins in the living cell (13). For example, phosphorylation and glycosylation induce conformational changes in target proteins, modulating their enzymatic activities and interactions with other molecules $(14,15)$. Protein biotinylation, lipoylation, and phosphopantetheinylation install crucial functional groups that directly participate in substrate turnover at the active site of biosynthetic enzymes (16-18). Protein lipidation specifies the subcellular localization of the modified target and its trafficking pathway within the cell (19). At the end of the protein lifespan, ubiquitination marks proteins for proteolytic degradation (20).

Since PTM regulates the structure, function, and interactions of the target proteins with other cellular entities, virtually all signaling events rely on tandem PTMs to transmit messages through dynamic signal transduction networks and to synchronize the processes of cell biology. This is evident in the regulation of p53 tumor-suppressor activity by phosphorylation of specific serine and threonine residues and by the acetylation and ubiquitination of lysine residues (21). It is also well-established that histone modification by phosphorylation, methylation, and acetylation affect chromatin structure and consequently transcription (22). Furthermore, PTM is responsible for the activation of many biosynthetic pathways, such as phosphopantetheinylation of fatty acid synthase, nonribosomal peptide synthetases (NRPS) and polyketide synthases (PKS), biotinylation of carboxylases, and lipoylation of $\alpha$-keto acid dehydrogenase (23). These enzymes play important roles in the biosynthesis of metabolites crucial for the normal function of the cell. Thus, profiling the catalytic activity of PTM enzymes and mapping the hierarchy of modification events would begin to elucidate regulation mechanisms essential for normal cell function, and set the stage for developing therapies to target the dysregulated cellular state.

\section{Profiling PTM Enzymes by Phage Display}

An immediate challenge for the systematic profiling of PTM enzymes is the complexity of the protein modification 


\section{Tech Insight}

cascade and the crosstalk between multiple signal transduction pathways - one PTM enzyme may have multiple downstream targets, and one specific PTM on the target protein may result from the combined action of multiple PTM enzymes from different pathways, further increasing the workload for genome-wide PTM enzyme profiling. To tackle this inherent complexity, high-throughput systems have been designed to identify the downstream targets of specific PTM enzymes. For example, various chemical genetics and proteomics methods have been developed for the screening of PTM activities $(24,25)$. Some methods take advantage of mechanism-based inhibitors or photocrosslinkers that label specific classes of enzymes with unique chemical functionalities (fluorescence or affinity probes) $(26,27)$. Others have exploited the substrate promiscuity of PTM enzymes to modify their downstream target proteins with biotinylated substrates or substrates that can be subsequently conjugated to biotin. In these systems, biotin serves as an affinity handle with which the downstream targets of PTM enzymes can be identified $(28,29)$. In addition, protein or peptide microarrays have been developed as a high-throughput platform for profiling the activities of specific PTM enzymes (30).

So far, these methods have achieved numerous successes in identifying new PTM enzymes and their substrates, expanding our knowledge of protein regulation networks. However, since the cell proteome is directly profiled, target proteins with higher expression levels are easier to identify than those less abundant. Due to the sensitivity of these techniques, posttranslationally modified proteins present in low copy numbers tend to be overlooked.

In comparison, phage display provides a high-throughput selection system that allows target clones to be enriched by several orders of magnitude through iterative rounds of selection. This significantly increases the likelihood of identifying novel or scarce targets of PTM enzymes. The substrate phage display platform can exploit two strategies for identifying targets of PTM enzymes after the modification of a phage-displayed protein library. One system uses an antibody or a protein receptor that specifically binds modified proteins to select for the downstream targets of a PTM enzyme. A parallel platform uses immobilized streptavidin to capture phage-displayed protein targets that are modified with biotin-conjugated small molecule probes. In the following section, we describe an efficient application of phage display for profiling the downstream targets of phosphopantetheinyl transferase (PPTase) (12).

\section{Profiling PPTase Activity by Phage Display}

PPTase installs the 4'-phosphopantetheinyl (Ppant) group of coenzyme A ( $\mathrm{CoA})$ on a conserved Ser residue in the peptidyl carrier proteins (PCP) in NRPS or acyl carrier proteins (ACP) in PKS. This transfer of the Ppant moiety converts NRPS and PKS from their inactive apo forms into biologically functional holo forms, which allows for the biosynthesis of polyketide and nonribosomal peptide products, respectively (17). Since many polyketide and nonribosomal peptide molecules have therapeutic value as anticancer (epothilone, bleomycin) and antibiotic (vancomycin, erythromycin) agents, we were interested in developing a high-throughput method for the profiling of PKS and NRPS gene clusters. Such a method would accelerate the cloning of PKS and NRPS clusters from genomeunsequenced bacteria, lending access to the natural products synthesized by environmental microbes that currently cannot be cultured.

In Bacillus subtilis, the modification of carrier proteins in PKS or NRPS clusters by CoA is accomplished by Sfp, a PPTase capable of transferring a variety of small moleculePpant groups to the carrier protein from their CoA conjugates (31). For example, biotin can be conjugated to CoA through thioether or disulfide linkages and covalently loaded
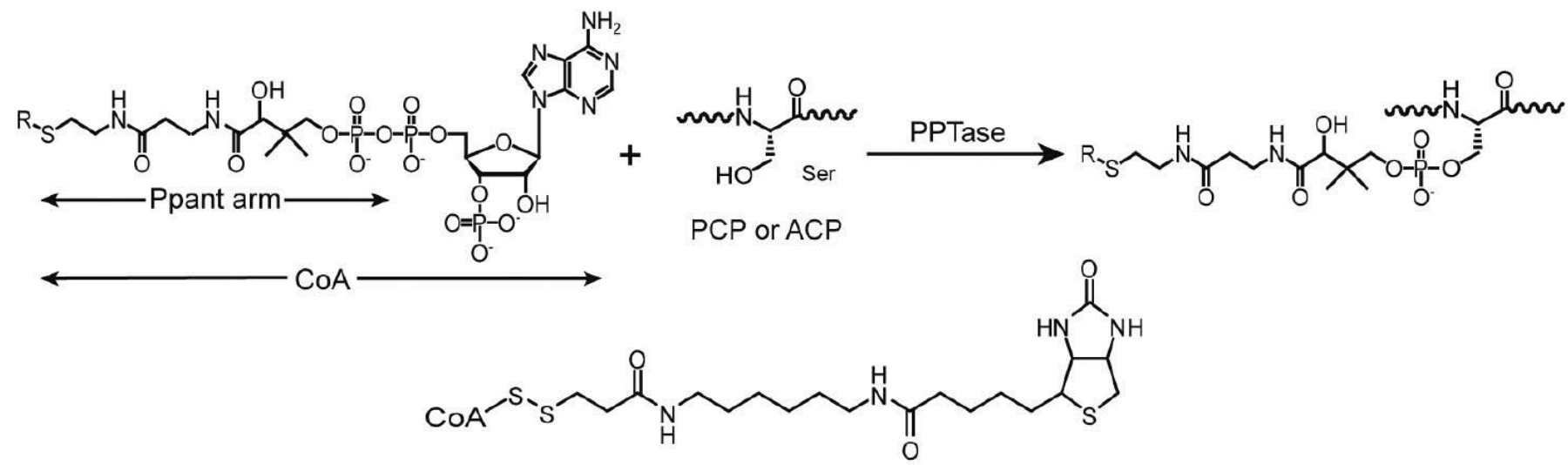

Biotin-SS-CoA (1)

Figure 1. Phosphopantetheinyl transferase (PPTase)-catalyzed 4'-phosphopantetheinyl (Ppant) group transfer to a conserved Ser residue in peptidyl carrier proteins (PCP) or acyl carrier proteins (ACP). The structure of biotin-SS-coenzyme A (CoA) (1) used in the selection is also shown. 


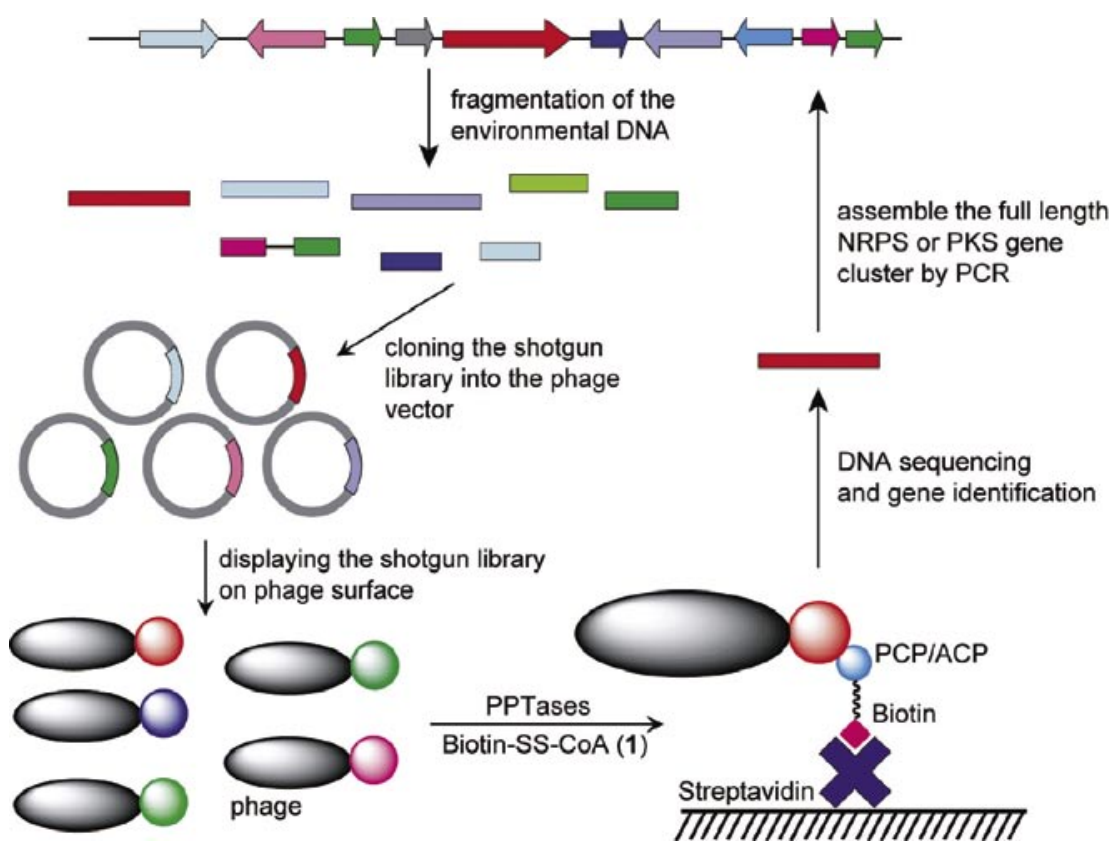

Figure 2. High-throughput cloning of nonribosomal peptide synthetases (NRPS) and polyketide synthases (PKS) biosynthetic gene clusters from shotgun environmental DNA libraries by phage display.

onto the carrier proteins embedded in the gene clusters of a diverse group of bacterial species (32). Based on the unique enzymatic activity of Sfp, we recently developed a technique for the efficient selection of carrier protein genes from a bacterial genomic library by M13 phage display (12). In this method, a shotgun genomic library was displayed on phage surface, and Sfp was used to covalently modify phage-displayed carrier proteins with biotin in presence of biotin-SS-CoA (Figure 1). Biotinylated phage particles were then selected by binding to immobilized streptavidin, and the corresponding NRPS or PKS genes encoding the carrier proteins were identified by DNA sequencing (Figure 2). Using this strategy, about one-half of the carrier proteins in $B$. subtilis, and one-fifth of the carrier proteins in Myxococcus xanthus were identified from the genomic DNA libraries with iterative rounds of selection (12).

The same phage-display method can potentially be used for the high-throughput cloning of PKS and NRPS biosynthetic gene clusters from genome-unsequenced bacteria or from environmental DNA samples. Shotgun environmental DNA libraries can be displayed on the phage surface, allowing clones displaying fragments of PKS and NRPS clusters to be selected for. After selection, corresponding full-length biosynthetic gene clusters can then be assembled by PCR based on the DNA sequence of the PKS or NRPS fragments identified by phage selection (Figure 2). Following assembly of full-length clusters, standard molecular cloning techniques could be applied to introduce the clusters into amenable host bacteria to allow synthesis of natural products and biological activity characterization.
Such a strategy would bypass the current barrier of having to isolate a bacterium in culture in order to characterize the natural product molecules it produces, opening the doors to the rich array of natural products offered by unculturable microbes.

\section{Other Phage-Display Systems for the Profiling of PTM Enzymes}

Following a similar strategy, short peptide substrates of biotin ligase and PPTase have been identified from randomized peptide libraries for the development of peptide tags for site-specific protein labeling by PTM enzymes $(3,33)$. Phage display has also been used to identify short peptide substrates of transglutaminase based upon affinity selection on a peptide library. The endogenous targets of transglutaminase were then identified by mapping these synthetic peptides onto the proteome $(34,35)$. Similar approaches have been developed to probe the substrate specificity of proteases based upon the proteolytic release of phage particles from a solid support. Methods also exist for profiling the substrate specificities of kinases by subjecting a phage-displayed peptide library for phosphorylation and selecting phosphorylated peptides using an antibody directed against phosphotyrosine (36).

Substrate phage display has been validated as a useful tool for identifying the downstream targets of a posttranslational modification enzyme in the proteome. Yet, this method only tackles half of the puzzle for the mapping of PTM networks. The question of how to identify the upstream enzymes responsible for modifying a target protein still remains. We should continue to integrate genomic, proteonomic, chemi$\mathrm{cal}$, and biochemical strategies in order to generate the molecular roadmap used by the PTM system for the regulation of fundamental biological processes.

\section{Acknowledgments}

We are thankful for a startup research fund provided by the University of Chicago and the Camille and Henry Dreyfus Foundation New Faculty Award to J.Y.

\section{References}

1. Smith, G.P. and V.A. Petrenko. 1997. Phage Display. Chem. Rev. 97:391-410.

2. Zozulya, S., M. Lioubin, R.J. Hill, C. Abram, and M.L. Gishizky. 1999. Mapping signal transduction pathways by phage display. Nat. Biotechnol. 17:1193-1198.

3. Beckett, D., E. Kovaleva, and P.J. Schatz. 1999. A minimal peptide substrate in biotin holoenzyme synthetase-catalyzed 
biotinylation. Protein Sci. 8:921-929.

4. Yin, J., P.D. Straight, S.M. McLoughlin, Z. Zhou, A.J. Lin, D.E. Golan, N.L. Kelleher, R. Kolter, and C.T. Walsh. 2005. Genetically encoded short peptide tag for versatile protein labeling by Sfp phosphopantetheinyl transferase. Proc. Natl. Acad. Sci. USA 102:15815-15820.

5. Cesaro-Tadic, S., D. Lagos, A. Honegger, J.H. Rickard, L.J. Partridge, G.M. Blackburn, and A. Pluckthun. 2003. Turnoverbased in vitro selection and evolution of biocatalysts from a fully synthetic antibody library. Nat. Biotechnol. 21:679-685.

6. Demartis, S., A. Huber, F. Viti, L. Lozzi, L. Giovannoni, P. Neri, G. Winter, and D. Neri. 1999. A strategy for the isolation of catalytic activities from repertoires of enzymes displayed on phage. J. Mol. Biol. 286:617-633.

7. Janda, K.D., L.C. Lo, C.H. Lo, M.M. Sim, R. Wang, C.H. Wong, and R.A. Lerner. 1997. Chemical selection for catalysis in combinatorial antibody libraries. Science 275:945-948.

8. Pedersen, H., S. Holder, D.P. Sutherlin, U. Schwitter, D.S. King, and P.G. Schultz. 1998. A method for directed evolution and functional cloning of enzymes. Proc. Natl. Acad. Sci. USA 95:10523-10528.

9. Xia, G., L. Chen, T. Sera, M. Fa, P.G. Schultz, and F.E. Romesberg. 2002. Directed evolution of novel polymerase activities: mutation of a DNA polymerase into an efficient RNA polymerase. Proc. Natl. Acad. Sci. USA 99:6597-6602.

10. Yin, J., J.H. Mills, and P.G. Schultz. 2004. A catalysis-based selection for peroxidase antibodies with increased activity. J. Am. Chem. Soc. 126:3006-3007.

11. Burch, L.R., M. Scott, E. Pohler, D. Meek, and T. Hupp. 2004. Phage-peptide display identifies the interferon-responsive, death-activated protein kinase family as a novel modifier of MDM2 and p21WAF1. J. Mol. Biol. 337:115-128.

12. Yin, J., P.D. Straight, S. Hrvatin, P.C. Dorrestein, S.B. Bumpus, C. Jao, N.L. Kelleher, R. Kolter, and C.T. Walsh. 2007. Genome-wide high-throughput mining of natural-product biosynthetic gene clusters by phage display. Chem. Biol. 14:303-312.

13. Walsh, C.T., S. Garneau-Tsodikova, and G.J. Gatto, Jr. 2005. Protein posttranslational modifications: the chemistry of proteome diversifications. Angew. Chem. Int. Ed. Engl. 44:73427372.

14. Johnson, L.N. and R.J. Lewis. 2001. Structural basis for control by phosphorylation. Chem. Rev. 101:2209-2242.

15.Zachara, N.E. and G.W. Hart. 2002. The emerging significance of O-GIcNAc in cellular regulation. Chem. Rev. 102:431438.

16. Chapman-Smith, A. and J.E. Cronan, Jr. 1999. The enzymatic biotinylation of proteins: a post-translational modification of exceptional specificity. Trends Biochem. Sci. 24:359-363.

17. Lambalot, R.H., A.M. Gehring, R.S. Flugel, P. Zuber, M. LaCelle, M.A. Marahiel, R. Reid, C. Khosla, and C.T. Walsh. 1996. A new enzyme superfamily - the phosphopantetheinyl transferases. Chem. Biol. 3:923-936.

18.Zhao, X., J.R. Miller, Y. Jiang, M.A. Marletta, and J.E. Cronan. 2003. Assembly of the covalent linkage between lipoic acid and its cognate enzymes. Chem. Biol. 10:1293-1302.

19. Resh, M.D. 2006. Trafficking and signaling by fatty-acylated and prenylated proteins. Nat. Chem. Biol. 2:584-590.

20.Pickart, C.M. 2001. Mechanisms underlying ubiquitination. Annu. Rev. Biochem. 70:503-533.

21. Bode, A.M. and Z. Dong. 2004. Post-translational modification of p53 in tumorigenesis. Nat. Rev. Cancer 4:793-805.

22. Strahl, B.D. and C.D. Allis. 2000. The language of covalent histone modifications. Nature 403:41-45.

23.Perham, R.N. 2000. Swinging arms and swinging domains in multifunctional enzymes: catalytic machines for multistep reactions. Annu. Rev. Biochem. 69:961-1004.

24.Cook, B.N. and C.R. Bertozzi. 2002. Chemical approaches to the investigation of cellular systems. Bioorg. Med. Chem. 10:829-840.

25. Speers, A.E. and B.F. Cravatt. 2004. Chemical strategies for activity-based proteomics. ChemBioChem 5:41-47.

26.Kho, Y., S.C. Kim, C. Jiang, D. Barma, S.W. Kwon, J. Cheng, J. Jaunbergs, C. Weinbaum, et al. 2004. A tagging-via-substrate technology for detection and proteomics of farnesylated proteins. Proc. Natl. Acad. Sci. USA 101:12479-12484.

27. Saghatelian, A., N. Jessani, A. Joseph, M. Humphrey, and B.F. Cravatt. 2004. Activity-based probes for the proteomic profiling of metalloproteases. Proc. Natl. Acad. Sci. USA 101:10000-10005.

28. Khidekel, N., S.B. Ficarro, E.C. Peters, and L.C. Hsieh-Wilson. 2004. Exploring the O-GlcNAc proteome: direct identification of O-GIcNAc-modified proteins from the brain. Proc. Natl. Acad. Sci. USA 101:13132-13137.

29. Vocadlo, D.J., H.C. Hang, E.J. Kim, J.A. Hanover, and C.R. Bertozzi. 2003. A chemical approach for identifying OGlcNAc-modified proteins in cells. Proc. Natl. Acad. Sci. USA 100:9116-9121.

30.Pokholok, D.K., C.T. Harbison, S. Levine, M. Cole, N.M. Hannett, T.I. Lee, G.W. Bell, K. Walker, et al. 2005. Genome-wide map of nucleosome acetylation and methylation in yeast. Cell 122:517-527.

31. Yin, J., A.J. Lin, D.E. Golan, and C.T. Walsh. 2006. Site-specific protein labeling by Sfp phosphopantetheinyl transferase. Nat. Protoc. 1:280-285.

32.La Clair, J.J., T.L. Foley, T.R. Schegg, C.M. Regan, and M.D. Burkart. 2004. Manipulation of carrier proteins in antibiotic biosynthesis. Chem. Biol. 11:195-201.

33.Zhou, Z., P. Cironi, A.J. Lin, Y. Xu, S. Hrvatin, D.E. Golan, P.A. Silver, C.T. Walsh, and J. Yin. 2007. Genetically encoded short peptide tags for orthogonal protein labeling by Sfp and AcpS phosphopantetheinyl transferases. ACS Chem Biol. 2:337346.

34.Keresztessy, Z., E. Csosz, J. Harsfalvi, K. Csomos, J. Gray, R.N. Lightowlers, J.H. Lakey, Z. Balajthy, and L. Fesus. 2006. Phage display selection of efficient glutamine-donor substrate peptides for transglutaminase 2. Protein Sci. 15:2466-2480.

35.Sugimura, Y., M. Hosono, F. Wada, T. Yoshimura, M. Maki, and K. Hitomi. 2006. Screening for the preferred substrate sequence of transglutaminase using a phage-displayed peptide library: identification of peptide substrates for TGASE 2 and Factor XIIIA. J. Biol. Chem. 281:17699-17706.

36.Deng, S.J., W. Liu, C.A. Simmons, J.T. Moore, and G. Tian. 2001. Identifying substrates for endothelium-specific Tie2 receptor tyrosine kinase from phage-displayed peptide libraries for high throughput screening. Comb. Chem. High Throughput Screen. 4:525-533. 